\title{
Linear theory of particulate Rayleigh-Bénard instability
}

\author{
Suryansh Prakhar $\odot^{1, *}$ and Andrea Prosperetti ${ }^{1,2,3, \dagger}$ \\ ${ }^{1}$ Department of Mechanical Engineering, University of Houston, 4726 Calhoun Road, Houston, \\ Texas 77204-4006, USA \\ ${ }^{2}$ Faculty of Science and Technology and J. M. Burgers Centre for Fluid Dynamics, University of Twente, \\ P.O. Box 217, 7500 AE Enschede, Netherlands \\ ${ }^{3}$ Department of Mechanical Engineering, Johns Hopkins University, Baltimore, Maryland 21218, USA
}

(Received 22 May 2021; accepted 26 July 2021; published 11 August 2021)

\begin{abstract}
A two-fluid model is used to study the effect of point particles on the Rayleigh-Bénard stability threshold in a laterally unbounded cell. Equal particles are steadily and uniformly introduced at the top plate at their terminal velocity with a fixed temperature. Both velocity and temperature are allowed to vary while, falling, the particles interact with the fluid. This interaction is modulated by the ratio of the particle density and heat capacity to those of the fluid. Particles are found to have a stabilizing effect, which increases with their concentration and density up to several orders of magnitude above the single-phase stability threshold. This result is primarily a consequence of the particle mechanical, rather than thermal, coupling with the fluid. The particle initial temperature has a strong effect on the undisturbed temperature distribution in the cell, which is a significant factor for the stability of the system. The addition of particles greatly increases the dimension of the parameter space necessary to characterize the flow.
\end{abstract}

DOI: 10.1103/PhysRevFluids.6.083901

\section{INTRODUCTION}

The importance of particulate flows in nature and technology has motivated a rich literature devoted to improving our understanding of the physical processes that determine their behavior. The major part of this work has dealt with isothermal systems (see, e.g., [1-3] for recent reviews), but study of the effect of suspended particles on heat transfer processes - and, in particular, on Rayleigh-Bénard convection, which is the topic of the present paper-is gaining importance.

A significant impulse to this line of research was given by interest in the heat transport properties of so-called nanofluids. In a series of papers, Nield and Kuznetsov studied natural convection of nanofluids in different situations [4-7] on the basis of the mathematical model proposed in Ref. [8]. This and related work is reviewed in [9-11], among others. Nanoparticles are sensitive to processes, such as Brownian motion and thermophoresis, which quickly become unimportant as the particle size grows to the micrometer scale and beyond. In this size range particle inertia becomes significant and the homogeneous-fluid model often adopted for nanofluids is no longer appropriate.

There are a few theoretical studies dealing with particle-resolved simulations of particulate Rayleigh-Bénard convection (see, e.g., [12-16]), but much of the work on this topic has relied on extensions of the Lagrangian-Eulerian point-particle model widely used in the study of adiabatic turbulence in particulate flow. References $[17,18]$ studied the effect on the Nusselt number of particles

\footnotetext{
"Present address: Department of Mechanical Engineering, Johns Hopkins University, Baltimore MD 21218, USA; suryansh@outlook.in

†aprosper@central.uh.edu
} 
introduced at the upper plate of a cylindrical Rayleigh-Bénard cell at a weakly turbulent Rayleigh number of $2 \times 10^{6}$. The particle-fluid mechanical coupling was found to be more significant than the thermal coupling in affecting the heat transfer through the cell. This conclusion was also reached in Ref. [19] at the same Rayleigh number. The Nusselt number was found to increase with the particle loading and the particle specific heat reaching a maximum for a particle Stokes number of about 1 . This effect occurs in spite of an attenuation of the turbulent kinetic energy and is due to the direct transport of thermal energy by the particles which are picked up by the turbulent eddies near the hot bottom plate and carried up by the rising plumes. When the Rayleigh number is increased up to $10^{12}$ the situation becomes more complex [20]. At such large Rayleigh numbers there are large-scale circulating structures that carry the particle for a long time hindering their settling. Furthermore, particles do not settle uniformly on the base of the cell but preferentially under regions of large upwelling. A very recent study focuses on micron-sized particles and describes implication for the spreading of pollutants and contaminants [21]. Other situations have been studied theoretically by similar means. References [22-24] studied particle transport and settling in a differentially heated cavity at Rayleigh number up to $7 \times 10^{8}$ using one-way and two-way coupling. Reference [25] studied the transport of particles in a laminar thermal plume. Buoyancy effects caused by the direct heating of the particles have been investigated in the context of solar energy harvesting (see, e.g., [26-28]).

The abundance of studies devoted to effects of buoyancy in particulate flows that can be deduced from these examples is a witness to the practical importance of these phenomena in nature (e.g., crystallization in magma chambers, snow and rain formation), technology (e.g., metallurgy, fluidized beds, solar energy), and society (e.g., dispersion of pollutants). Motivated by these applications, this paper presents a study of the linear stability of a laterally unbounded particulate Rayleigh-Bénard system based on a standard two-fluid model. Particles are assumed to be introduced steadily and uniformly at the upper plate and to be removed at the bottom plate. The downward motion of the particles renders the spatial operator of the problem non-self-adjoint so that the principle of exchange of stabilities prevailing in the single-phase version of the problem (see, e.g., [29]) does not apply and some of the eigenvalues are complex. The numerical solution of the eigenvalue problem shows however that the eigenvalues with the largest real part, on which the stability of the system depends, are real. Near marginal conditions, the complex eigenvalues have a negative real part and do not affect the stability of the system.

The mathematical setup that we develop has some similarities with Rayleigh-Bénard convection in a porous medium which has been studied extensively (see, e.g., [30-32]), both in connection with geothermal energy and geological sequestration of carbon dioxide (see, e.g., [33-36]), and in view of its intrinsic scientific interest (see, e.g., [37-40]).

\section{MATHEMATICAL MODEL}

One of the basic frameworks for the study of particulate flows is the so-called point-particle model in which the finite size of the particles is disregarded. This model goes back to the early days of the study of these systems (see, e.g., [41-43]) and has been widely used ever since (see, e.g., $[1,3,44,45])$. Its use is justified when the fluid-particles mixture is dilute and the particles are much smaller than all the flow length scales. In the Eulerian-Lagrangian version of the point-particle model, the effect of the particles is included in the fluid equations as a sum of delta functions centered at the particle positions multiplied by appropriate sources, i.e., forces for the momentum equation and energies for the energy equation (see, e.g., [1,44]). The position, velocity, and energy of the particles, on the other hand, are found by integrating the momentum and energy equations for each particle. It is not straightforward to adapt this framework to the study of the linear stability of the Rayleigh-Bénard problem, while an Eulerian-Eulerian description, with quantities pertaining to both the fluid and the particulate phase described by continuous fields, considerably simplifies the task. Conceptually, this latter description can be connected to the Eulerian-Lagrangian one by replacing the sum over the sources representing the contribution of the particle contained in 
a macroscopically small volume of the mixture by the product of the number of contributing particles multiplied by average values of the sources. With the Boussinesq approximation (see, e.g., $[29,31,46])$, the fluid momentum equation modified to include the effect of the particles is then given by

$$
\rho_{f}\left(\partial_{t} \mathbf{u}+\mathbf{u} \cdot \nabla \mathbf{u}\right)=-\nabla p+\mu_{f} \nabla^{2} \mathbf{u}+\rho_{f}\left[1-\beta\left(T-T_{r}\right)\right] \mathbf{g}-n m_{p} \mathbf{f} .
$$

Here $\mathbf{u}, p$, and $T$ are the fluid velocity, pressure, and temperature fields, $\mu_{f}, \beta$, and $\rho_{f}$ are the fluid viscosity, thermal expansion coefficient and density at the reference temperature $T_{r}$, and $\mathbf{g}$ is the (vector) acceleration of gravity. The particles are assumed to be equal spheres with mass $m_{p}$, density $\rho_{p}$, and diameter $d_{p}$, and to be distributed with a local number density $n$. The force per unit particle mass exerted by the fluid on the particles is denoted by $\mathbf{f}$. We model this quantity in a fairly standard way as

$$
\mathbf{f}=\frac{\mathbf{u}-\mathbf{w}}{\tau_{p}}
$$

in which $\mathbf{w}$ is the velocity field of the particulate phase and $\tau_{p}$ is the particle characteristic time given by

$$
\tau_{p}=\frac{\rho_{p} d_{p}^{2}}{18 \rho_{f} v_{f} r_{\mathrm{Re}}},
$$

with $v_{f}=\mu_{f} / \rho_{f}$ the fluid kinematic viscosity. The factor $r_{\mathrm{Re}}$, defined by

$$
r_{\mathrm{Re}}=1+0.15 \mathrm{Re}_{p}^{0.687}
$$

represents a finite-Reynolds-number correction to the Stokes drag force (see, e.g., [47]); the particle Reynolds number $\operatorname{Re}_{p}$ is defined later in (2.15). Consistently with the point-particle approximation, the particle effect on the fluid continuity equation is neglected so that

$$
\nabla \cdot \mathbf{u}=0
$$

In a field framework, the ordinary time derivative following each particle of the EulerianLagrangian description is replaced by a convective derivative so that conservation of the particle number density is expressed by

$$
\partial_{t} n+\nabla \cdot(n \mathbf{w})=0
$$

while the momentum equation for the particulate phase is

$$
\partial_{t} \mathbf{w}+\mathbf{w} \cdot \nabla \mathbf{w}=\mathbf{f}+\left(1-\frac{\rho_{f}}{\rho_{p}}\right) \mathbf{g},
$$

in which the last term represents the particle weight corrected for the buoyancy force.

Similarly to (2.1), the fluid energy equation is written as

$$
\partial_{t} T+\mathbf{u} \cdot \nabla T=D_{f} \nabla^{2} T+n \frac{Q_{p}}{\rho_{f} c_{p f}},
$$

with $c_{p f}$ the fluid specific heat and $Q_{p}$ the heat transferred by the particles to the fluid, for which we assume the form

$$
Q_{p}=\pi d_{p}^{2} h_{p}\left(T_{p}-T\right)
$$

in which $T_{p}$ is the temperature field of the particulate phase. The heat transfer coefficient $h_{p}$ is expressed in terms of the Nusselt number $\mathrm{Nu}_{p}$, for which we use a standard correlation applicable to spheres, namely (see, e.g., [47-49]),

$$
\mathrm{Nu}_{p}=\frac{d_{p} h_{p}}{k_{f}}=2+0.6 \operatorname{Re}_{p}^{1 / 2} \operatorname{Pr}^{1 / 3},
$$


with $k_{f}$ the fluid thermal conductivity and Pr the fluid Prandtl number. The particle temperature is treated in the lumped-capacitance approximation so that the energy equation for the particulate phase is

$$
m_{p} c_{p p}\left(\partial_{t} T_{p}+\mathbf{w} \cdot \nabla T_{p}\right)=-Q_{p},
$$

with $c_{p p}$ the specific heat of the particle material. On the basis of this equation we can define a temperature relaxation time for the particles similar to (2.3), namely

$$
\tau_{\mathrm{th}}=\frac{m_{p} c_{p p}}{\pi d_{p}^{2} h_{p}}
$$

in terms of which (2.11) becomes

$$
\partial_{t} T_{p}+\mathbf{w} \cdot \nabla T_{p}=\frac{T-T_{p}}{\tau_{\mathrm{th}}} .
$$

\section{A. Unperturbed state}

We assume a base state in which the fluid is at rest and the particles are uniformly distributed with a number density $n_{0}$ and fall at their terminal velocity $w_{0}$ given, from (2.7), by

$$
\mathbf{w}_{0}=\left(1-\frac{\rho_{f}}{\rho_{p}}\right) \tau_{p} \mathbf{g} \equiv-w_{0} \mathbf{k},
$$

with $\mathbf{k}$ a unit vector in the $z$ direction directed upward against gravity. The particle Reynolds number in terms of this velocity is given by

$$
\operatorname{Re}_{p}=\frac{d_{p} w_{0}}{v_{f}}=\frac{\left(\rho_{p} / \rho_{f}-1\right) g d_{p}^{3}}{18 v_{f}^{2} r_{\operatorname{Re}}} .
$$

As discussed in Ref. [18] if, at the upper boundary, particles were introduced with zero velocity and allowed to accelerate to their terminal velocity, a dense, nonuniform particle layer would form which would obscure the interpretation of the stability results. To avoid this inessential complication, we assume that particles are introduced at their terminal velocity. This procedure is equivalent to assuming that the distance traveled by the particles during the acceleration phase, of the order of $w_{0} \tau_{p}$, is much smaller than the cell height. Accumulation of particles at the lower boundary is also assumed to be a negligible effect. This aspect of the model may be justified if the particle-fluid mixture is dilute and the particle size is much smaller than the cell height so that the thickness of the particle layer grows very slowly essentially maintaining the temperature of the bottom plate. Both assumptions are satisfied when reliance on the point-particle model is justified. It may also be noted that a similar device to avoid the inessential effects introduced by the boundary layers that form near the plates is adopted in the so-called "homogeneous models" of single-phase Rayleigh-Bénard convection in which the top- and bottom-temperature boundary conditions are replaced by periodic ones, with a temperature gradient imposed (see, e.g., [50,51]).

The undisturbed fluid pressure field corresponding to the situation envisaged is found from (2.1) as

$$
\nabla p_{0}=\rho_{f}\left[1+\beta\left(T_{0}-T_{r}\right)\right] \mathbf{g}+\frac{m_{p} n_{0}}{\tau_{p}} \mathbf{w}_{0},
$$

in which $T_{0}=T_{0}(z)$ is the undisturbed temperature field in the fluid. It is evident from this result that horizontal variations of the undisturbed particle number density $n_{0}$ or fluid temperature $T_{0}$ would induce horizontal variations of the pressure field which, in turn, would give rise to fluid flow. We return on this point in Sec. II C.

A consideration of the energy equations leads to the identification of two characteristic length scales which play an important role in what follows. In the first place, the steady version of the 
particulate energy equation (2.11) may be written as

$$
T_{0}=-\ell \partial_{z} T_{p 0}+T_{p 0},
$$

in which $T_{p 0}=T_{p 0}(z)$ is the temperature field of the particulate phase in the unperturbed base state. The length $\ell$, defined by

$$
\ell=\frac{m_{p} c_{p p} w_{0}}{\pi d_{p}^{2} h_{p 0}}
$$

characterizes the distance traversed by a particle during the time necessary for its temperature to equalize with that of the surrounding fluid. This interpretation is justified by noting that, if $\Delta T$ is a typical fluid-particle temperature difference, $\left(m_{p} c_{p p} \Delta T\right) /\left(\pi d_{p}^{2} h_{p 0} \Delta T\right)$ is the ratio of the energy content of the particle to the rate at which this energy is transmitted to the fluid, so that $\ell$ equals the particle fall velocity $w_{0}$ multiplied by this timescale. Second, upon eliminating $Q_{p}$ between the two energy equations (2.8) and (2.11), we find

$$
\partial_{z}^{2} T_{0}+\frac{\ell}{L^{2}} \partial_{z} T_{p 0}=0
$$

in which

$$
L=\sqrt{\frac{k_{f}}{\pi d_{p}^{2} h_{p} n_{0}}} \sim \sqrt{\frac{k_{f} \Delta T}{n_{0} Q_{p}}},
$$

with $\Delta T$ a typical temperature difference as before. To elucidate the physical meaning of this length scale we rewrite (2.19) in order of magnitude as

$$
\Delta q_{f} \sim \frac{\ell}{L^{2}} k_{f} \partial_{z} T_{p 0}
$$

in which $\Delta q_{f}$ is the difference between the conduction heat fluxes in the fluid over a distance $L$ (which would of course vanish in the absence of the particles). Since $L / \ell$ is the number of layers over which the fluid and particle temperatures equalize, this relation states that the change of $q_{f}$ equals the mean heat flux induced by the particle temperature gradient. In other words, $L$ is the characteristic distance over which the heat conduction in the fluid is significantly affected by the particles. This interpretation is also supported by rewriting (2.20) as

$$
A k_{f} \frac{\Delta T}{L} \sim A L n_{0} Q_{p},
$$

in which $A$ is an arbitrary horizontal area. The left-hand side is the heat flow rate in the fluid through the area $A$ while the right-hand side is the number of particles in a layer of thickness $L$, each one exchanging heat with the liquid at the rate $Q_{p}$.

Upon elimination of $T_{0}$ between (2.17) and (2.19) we have

$$
\partial_{z}\left(-\ell \partial_{z}^{2} T_{p 0}+\partial_{z} T_{p 0}+\frac{\ell}{L^{2}} T_{p 0}\right)=0
$$

We assume that the particles are injected with the temperature $T_{p t}$ at the top plate $z=\frac{1}{2} H$, with $H$ the height of the cell. The solution of this equation may then be written as

$$
T_{p 0}(z)=T_{p t}+K_{1}\left[1-e^{-k_{1}(H / 2-z)}\right]+K_{2}\left[1-e^{-k_{2}(H / 2-z)}\right],
$$

with $K_{1}$ and $K_{2}$ integration constants and

$$
k_{1,2}=\frac{1}{2 \ell}\left(1 \pm \sqrt{1+4 \frac{\ell^{2}}{L^{2}}}\right) .
$$




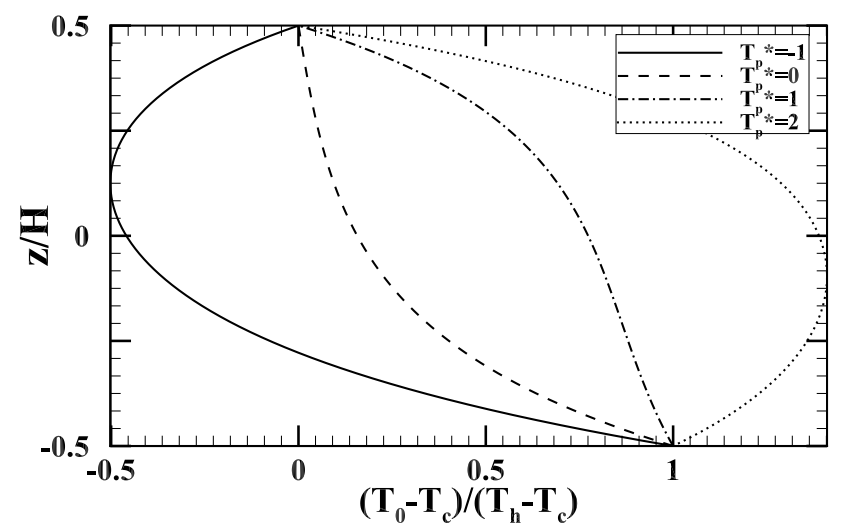

FIG. 1. The normalized undisturbed temperature distribution in the cell $\left[T_{0}(z / H)-T_{c}\right] /\left(T_{h}-T_{c}\right)$ for, from left to right, $\left(T_{p t}-T_{c}\right) /\left(T_{h}-T_{c}\right)=-1,0,1$, and 2 . The particle volume fraction is $\alpha_{0}=10^{-4}$, the dimensionless particle diameter $\delta=0.01$, and the particle Reynolds number $\operatorname{Re}_{p}=1$; the parameters $R, E$, and Pr have the same values as in Fig. 2 below.

From (2.17) we then find the temperature field in the fluid phase as

$$
T_{0}(z)=T_{p t}+K_{1}\left[1-\left(1-\ell k_{1}\right) e^{-k_{1}(H / 2-z)}\right]+K_{2}\left[1-\left(1-\ell k_{2}\right) e^{-k_{2}(H / 2-z)}\right] .
$$

The fluid temperature boundary conditions $T=T_{c}$ and $T=T_{h}$ at the top and bottom of the cell, $z= \pm \frac{1}{2} H$, determine the integration constants as

$$
\begin{gathered}
K_{1}=\frac{\left(T_{c}-T_{p t}\right)\left[1-\left(1-\ell k_{2}\right) e^{-k_{2} H}\right]-\ell k_{2}\left(T_{h}-T_{p t}\right)}{\ell k_{1}\left[1-\left(1-\ell k_{2}\right) e^{-k_{2} H}\right]-\ell k_{2}\left[1-\left(1-\ell k_{1}\right) e^{-k_{1} H}\right]}, \\
K_{2}=-\frac{\left(T_{c}-T_{p t}\right)\left[1-\left(1-\ell k_{1}\right) e^{-k_{1} H}\right]-\ell k_{1}\left(T_{h}-T_{p t}\right)}{\ell k_{1}\left[1-\left(1-\ell k_{2}\right) e^{-k_{2} H}\right]-\ell k_{2}\left[1-\left(1-\ell k_{1}\right) e^{-k_{1} H}\right]} .
\end{gathered}
$$

It can be checked that, for neutrally buoyant particles, for which $\ell=0$, the fluid temperature (2.26) reduces to the single-phase linear solution

$$
T_{0}=\frac{1}{2}\left(T_{h}+T_{c}\right)-\left(T_{h}-T_{c}\right) \frac{z}{H} .
$$

For future reference we show some examples of the normalized temperature profile $\left[T_{0}(z)-\right.$ $\left.T_{c}\right] /\left(T_{h}-T_{c}\right)$ in Fig. 1 for four values of the normalized particle injection temperature, $\left(T_{p t}-\right.$ $\left.T_{c}\right) /\left(T_{h}-T_{c}\right)=-1,0,1$, and 2 . The feature to note here is the major qualitative change of the temperature distribution and, in particular, of its gradient, which has such a profound effect on the stability problem. Very cold particles, with $\left(T_{p t}-T_{c}\right) /\left(T_{h}-T_{c}\right)=-1$, induce a large and negative gradient in the lower part of the cell, which is destabilizing. As the particle temperature increases past that of the cold plate, $\left(T_{p t}-T_{c}\right) /\left(T_{h}-T_{c}\right)=0$, and approaches that of the hot plate, $\left(T_{p t}-T_{c}\right) /\left(T_{h}-T_{c}\right)=1$, the gradient gradually decreases over a large portion of the cell. When the particles become even warmer, again a strong negative gradient appears, this time in the upper part of the cell, which will be seen to be destabilizing in certain ranges of the other parameters.

\section{B. Linearization}

We now linearize the mathematical model of the previous section by setting

$$
\begin{gathered}
\mathbf{u}=\mathbf{u}^{\prime}, \quad p=p_{0}+p^{\prime}, \quad T=T_{0}+T^{\prime}, \\
n=n_{0}+n^{\prime}, \quad \mathbf{w}=-w_{0} \mathbf{k}+\mathbf{w}^{\prime}, \quad T_{p}=T_{p 0}+T_{p}^{\prime},
\end{gathered}
$$


with the primed quantities assumed small with respect to the corresponding base-state fields carrying the index zero (for the fluid velocity smallness is understood in the sense that $\left|\partial_{t} \mathbf{u}^{\prime}\right| \gg\left|\mathbf{u}^{\prime} \cdot \nabla \mathbf{u}^{\prime}\right|$ ). For simplicity, we omit to perturb the drag force and the heat transfer coefficients evaluating them at base-state conditions. This slight inconsistency affects the particle mechanical relaxation time via the Reynolds-number correction $r_{\text {Re }}$ defined in (2.4) and the thermal relaxation time via the Nusselt number (2.10), neither of which depends strongly on $\mathrm{Re}_{p}$ in the range investigated in this paper.

After subtraction of the base-state relations, and omitting quadratic terms in the perturbed quantities, the fluid model equations become

$$
\begin{gathered}
\nabla \cdot \mathbf{u}^{\prime}=0 \\
\partial_{t} \mathbf{u}^{\prime}=-\frac{1}{\rho_{f}} \nabla p^{\prime}+v_{f} \nabla^{2} \mathbf{u}^{\prime}-\beta T^{\prime} \mathbf{g}+\frac{\rho_{p}}{\rho_{f}} n_{0} v_{p} \frac{\mathbf{w}^{\prime}-\mathbf{u}^{\prime}}{\tau_{p}}-\frac{m_{p} w_{0}}{\rho_{f} \tau_{p}} n^{\prime} \mathbf{k}, \\
\partial_{t} T^{\prime}+u_{z}^{\prime} \partial_{z} T_{0}=D_{f} \nabla^{2} T^{\prime}+\frac{Q_{p 0}}{\rho_{f} c_{p f}} n^{\prime}-\frac{\rho_{p} c_{p p} n_{0} v_{p}}{\rho_{f} c_{p f}} \frac{T^{\prime}-T_{p}^{\prime}}{\tau_{\text {th }}} .
\end{gathered}
$$

In (2.33) $v_{p}=\frac{\pi}{6} d_{p}^{3}$ is the particle volume and, in (2.34), $u_{z}^{\prime}$ is the vertical component of the fluid perturbation velocity. The corresponding equations for the particulate phase are

$$
\begin{gathered}
\partial_{t} n^{\prime}-w_{0} \partial_{z} n^{\prime}+n_{0} \nabla \cdot \mathbf{w}^{\prime}=0, \\
\partial_{t} \mathbf{w}^{\prime}-w_{0} \partial_{z} \mathbf{w}^{\prime}=\frac{\mathbf{u}^{\prime}-\mathbf{w}^{\prime}}{\tau_{p}}, \\
\partial_{t} T_{p}^{\prime}-w_{0} \partial_{z} T_{p}^{\prime}+\mathbf{w}^{\prime} \cdot \nabla T_{p 0}=\frac{T^{\prime}-T_{p}^{\prime}}{\tau_{\mathrm{th}}} .
\end{gathered}
$$

It is interesting to point out a similarity between the momentum equation (2.33) and the corresponding equation of the Brinkman model for flow in a fluid-saturated porous medium (see, e.g., [30,31]). Indeed, written out in detail, the term multiplying $\mathbf{w}^{\prime}-\mathbf{u}^{\prime}$ is

$$
\frac{\rho_{p} n_{0} v_{p}}{\rho_{f} \tau_{p}}=\frac{18 n_{0} v_{p} r_{\mathrm{Re}}}{d_{p}^{2}} v_{f} .
$$

The permeability $\kappa$ of a porous medium is defined in such a way that the pressure gradient equals $\left(-\mu_{f} / \kappa\right)$ times the (relative) velocity. For the present case in which the particles apply a point force to the fluid, this definition leads to

$$
\kappa=\frac{d_{p}^{2}}{18 n_{0} v_{p} r_{\mathrm{Re}}},
$$

which is precisely the inverse of the fraction in (2.38). A similar interpretation can be developed for the fraction multiplying $T^{\prime}-T_{p}^{\prime}$ in the energy equation (2.34), bringing this term to a form similar to the one adopted in the local thermal nonequilibrium model of heat transfer in fluid-saturated porous media. One difference is that, in a porous medium, the solid phase is stationary while, here, it is falling. This is not a major difference as long as the particles are uniformly distributed which, as explained in Sec. III below, is the situation explored in detail in this paper. Nevertheless, it may be noted that, in principle, a spatial particle nonuniformity can give a mechanical contribution to the instability of the system, an effect that is absent in a porous medium in which, at most, nonuniformities of the solid-phase distribution can only affect stability by thermal processes. Another difference is that, here, the particles can be introduced with an arbitrary temperature while, in a porous medium, the solid-phase temperature may lag that of the fluid, but is usually enslaved to it, at least in the absence of radiation or radioactive heating. As shown in Fig. 1, the undisturbed particle temperature can have a major effect on the fluid temperature distribution in the cell which, as will be shown below, has some interesting consequences for the stability of the system. 


\section{Boundary conditions}

We envisage a cell with a height $H$ and much larger dimensions (infinite in the limit) in the horizontal directions. Since the fluid is confined within the cell, its normal velocity on the horizontal boundaries must vanish. Standard conditions of prescribed temperatures at the boundaries are also assumed so that

$$
u_{z}^{\prime}=0 \quad \text { and } \quad T^{\prime}=0 \quad \text { at } \quad z= \pm \frac{1}{2} H .
$$

We consider the case of no-slip conditions at the boundaries so that, from the fluid continuity equation (see, e.g., [29]),

$$
\partial_{z} u_{z}^{\prime}=0, \quad \text { at } \quad z= \pm \frac{1}{2} H .
$$

These boundary conditions on the fluid fields are the same ones adopted in the linear stability theory of the single-phase Rayleigh-Bénard problem.

For the particulate phase, the present mathematical model can describe various situations, all compatible with the postulated uniform base state. For example, particles can be introduced at the upper plate with a small spatial nonuniformity, a small temperature nonuniformity, or both. As can be understood from the expression (2.16) of the undisturbed pressure field, in these situations one would expect to encounter fluid flow for any temperature difference so that the stability threshold $T_{h}-T_{c}$ would effectively vanish. As a matter of fact, with a horizontal particle number nonuniformity, the stability threshold may even turn negative. While these are interesting situations well deserving to be investigated, a natural first step in a study of particulate Rayleigh-Bénard convection is to consider how the fluid stability is affected by particles introduced uniformly with a uniform temperature. This is the problem on which we focus the analysis that follows. Thus, on the upper boundary at $z=\frac{1}{2} H$, we impose that

$$
T_{p}^{\prime}=0, \quad w_{z}^{\prime}=0,
$$

in which $w_{z}^{\prime}$ is the vertical component of the particle disturbance velocity field. No conditions can be imposed on the disturbance velocity and temperature fields at the lower boundary $z=-\frac{1}{2} H$. The values of $T_{p}^{\prime}$ and $w_{z}^{\prime}$ there will depend on the interaction of the falling particles with the fluid and must be found as part of the problem solution.

\section{SETUP FOR THE SOLUTION}

To solve the perturbation problem posed in the previous section we start by taking the double curl of the fluid momentum equation (2.33) and considering the $z$ component of the result which is, after using (2.32),

$$
\partial_{t} \nabla^{2} u_{z}^{\prime}=v_{f} \nabla^{4} u_{z}^{\prime}-\beta g\left[\partial_{z}^{2} T^{\prime}-\nabla^{2} T^{\prime}\right]-\frac{\rho_{p}}{\rho_{f}} \alpha_{0} \frac{\partial_{z}\left(\nabla \cdot \mathbf{w}^{\prime}\right)-\nabla^{2} w_{z}^{\prime}+\nabla^{2} u_{z}^{\prime}}{\tau_{p}}+\frac{m_{p} w_{0}}{\rho_{f} \tau_{p}}\left[\partial_{z}^{2} n^{\prime}-\nabla^{2} n^{\prime}\right] .
$$

We decompose the fields into horizontal normal modes each one of which is assumed to depend on the horizontal coordinates $(x, y)$ proportionally to a function $f(x, y)$ with the property that

$$
\left(\partial_{x}^{2}+\partial_{y}^{2}\right) f=-k^{2} f,
$$

with $k$ the modulus of a wave number in the horizontal plane. With this ansatz, $\nabla^{2}=\partial_{z}^{2}-k^{2}$ and the previous equation becomes

$\partial_{t}\left(\partial_{z}^{2}-k^{2}\right) u_{z}^{\prime}=v_{f}\left(\partial_{z}^{2}-k^{2}\right)^{2} u_{z}^{\prime}-\beta g k^{2} T^{\prime}-\frac{\rho_{p}}{\rho_{f}} \alpha_{0} \frac{\partial_{z}\left(\nabla \cdot \mathbf{w}^{\prime}\right)+\left(\partial_{z}^{2}-k^{2}\right)\left(u_{z}^{\prime}-w_{z}^{\prime}\right)}{\tau_{p}}+\frac{m_{p} w_{0}}{\rho_{f} \tau_{p}} k^{2} n^{\prime}$. 
Upon taking the divergence of the particle momentum equation we find, using (2.32),

$$
\partial_{t}\left(\nabla \cdot \mathbf{w}^{\prime}\right)-w_{0} \partial_{z}\left(\nabla \cdot \mathbf{w}^{\prime}\right)=-\frac{\nabla \cdot \mathbf{w}^{\prime}}{\tau_{p}} .
$$

This equation shows that, as the particles fall from higher levels with velocity $w_{0}, \nabla \cdot \mathbf{w}^{\prime}$ will tend to relax to zero on a timescale $\tau_{p}$. It is therefore evident that, if $\nabla \cdot \mathbf{w}^{\prime}=0$ at the upper boundary, $\boldsymbol{\nabla} \cdot \mathbf{w}^{\prime}$ will vanish for all times. An alternative way to reach the same conclusion is to note that, as is evident from Sec. II B, the spatial operator of the problem is independent of time and, therefore, the general solution will be the sum of terms having an exponential time dependence proportional to $e^{\eta t}$, with the $\eta$ 's eigenvalues of the spatial operator. For $\nabla \cdot \mathbf{w}^{\prime}$, each one of these terms, $W^{\prime}(\mathbf{x})$, say, satisfies the equation

$$
\eta W^{\prime}-w_{0} \partial_{z} W^{\prime}=-\frac{W^{\prime}}{\tau_{p}}
$$

with solution

$$
W^{\prime}(\mathbf{x})=W_{t}^{\prime}(x, y) \exp \left[-\left(\eta+\frac{1}{\tau_{p}}\right) \frac{\frac{1}{2} H-z}{w_{0}}\right],
$$

with $W_{t}^{\prime}(x, y)$ the boundary condition on $W^{\prime}$ at the top boundary $z=\frac{1}{2} H$. If $W_{t}^{\prime}$ is taken to vanish, $W^{\prime}$ will vanish for all times and all $z$ 's in agreement with the previous statement. With this choice, particle nonuniformities cannot develop in the bulk of the system, but can still be introduced as boundary conditions on the upper plate. Indeed, the perturbation number density mode corresponding to $W^{\prime}$ can be found from (2.35)

$$
\eta n^{\prime}-w_{0} \partial_{z} n^{\prime}+n_{0} W^{\prime}=0
$$

and is

$$
n^{\prime}(\mathbf{x})=\left\{n_{t}^{\prime}(x, y)+\tau_{p} n_{0} W_{t}^{\prime}\left[1-\exp \left(-\frac{\frac{1}{2} H-z}{\tau_{p} w_{0}}\right)\right]\right\} \exp \left[-\frac{\eta}{w_{0}}\left(\frac{1}{2} H-z\right)\right]
$$

with $n_{t}^{\prime}(x, y)$ the value of the $n^{\prime}$ mode at the top boundary. As anticipated in Sec. IIC, this work focuses on the effect on the stability threshold of particles introduced uniformly at the top plate at the same rate as in the base state. Thus, we take $n_{t}^{\prime}=0$ so that $n^{\prime}=0$ as well. It should be noted that, with these choices, particle clustering, and the attendant sweeping effect (see, e.g., [52]), is eliminated as a potential factor affecting the stability of the system. Situations in which $W^{\prime}$ and $n^{\prime}$ are nonzero require a separate investigation.

The problem is now reduced to four equations in the unknowns $u_{z}^{\prime}, w_{z}^{\prime}, T^{\prime}, T_{p}^{\prime}$ :

$$
\begin{gathered}
\partial_{t}\left(\partial_{z}^{2}-k^{2}\right) u_{z}^{\prime}=v_{f}\left(\partial_{z}^{2}-k^{2}\right)^{2} u_{z}^{\prime}-\beta g k^{2} T^{\prime}-\frac{\rho_{p} n_{0} v_{p}}{\rho_{f}} \frac{\left(\partial_{z}^{2}-k^{2}\right)\left(u_{z}^{\prime}-w_{z}^{\prime}\right)}{\tau_{p}}, \\
\partial_{t} w_{z}^{\prime}-w_{0} \partial_{z} w_{z}^{\prime}=\frac{u_{z}^{\prime}-w_{z}^{\prime}}{\tau_{p}}, \\
\partial_{t} T^{\prime}+u_{z}^{\prime} \partial_{z} T_{0}=D_{f}\left(\partial_{z}^{2}-k^{2}\right) T^{\prime}-\frac{\rho_{p} c_{p p} n_{0} v_{p}}{\rho_{f} c_{p f}} \frac{T^{\prime}-T_{p}^{\prime}}{\tau_{\mathrm{th}}} \\
\partial_{t} T_{p}^{\prime}-w_{0} \partial_{z} T_{p}^{\prime}+w_{z}^{\prime} \partial_{z} T_{p 0}=\frac{T^{\prime}-T_{p}^{\prime}}{\tau_{\mathrm{th}}} .
\end{gathered}
$$

It may be noted that (3.10) and (3.12) can be solved to express $w_{z}^{\prime}$ and $T_{p}^{\prime}$ in terms of $u_{z}^{\prime}$ and $T^{\prime}$, respectively, so that the particle fields can be eliminated from (3.9) and (3.11). The resulting system of integro-differential equations can be reduced to differential equations by an additional differentiation with respect to $z$. We do not take these steps as they do not seem to lead to a significant simplification of the mathematical problem. 


\section{Nondimensionalization and nondimensional quantities}

We nondimensionalize lengths in terms of the cell height $H$, velocities in terms of the free-fall velocity $v_{f}=\sqrt{\left.g \beta T_{h}-T_{c}\right) H}$, time in terms of $H / v_{f}$, and temperatures in terms of $T_{h}-T_{c}$. Upon so doing, Eqs. (3.9) to (3.12) become

$$
\begin{gathered}
\left(\partial_{z}^{2}-k^{2}\right)\left[\partial_{t} u_{z}^{\prime}-\sqrt{\frac{\operatorname{Pr}}{\operatorname{Ra}}}\left(\partial_{z}^{2}-k^{2}\right) u_{z}^{\prime}\right]+k^{2} T^{\prime}+\frac{\mathcal{M}}{\mathrm{St}_{m}}\left(\partial_{z}^{2}-k^{2}\right)\left(u_{z}^{\prime}-w_{z}^{\prime}\right)=0, \\
\partial_{t} w_{z}^{\prime}-\frac{\operatorname{Re}_{p}}{\delta} \sqrt{\frac{\operatorname{Pr}}{\operatorname{Ra}}} \partial_{z} w_{z}^{\prime}-\frac{u_{z}^{\prime}-w_{z}^{\prime}}{\mathrm{St}_{m}}=0 \\
\partial_{t} T^{\prime}+u_{z}^{\prime} \partial_{z} T_{0}-\frac{1}{\sqrt{\operatorname{Ra} \operatorname{Pr}}}\left(\partial_{z}^{2}-k^{2}\right) T^{\prime}+\frac{\mathcal{E}}{\mathrm{St}_{\mathrm{th}}}\left(T^{\prime}-T_{p}^{\prime}\right)=0 \\
\partial_{t} T_{p}^{\prime}-\frac{\operatorname{Re}_{p}}{\delta} \sqrt{\frac{\operatorname{Pr}}{\operatorname{Ra}}} \partial_{z} T_{p}^{\prime}+w_{z}^{\prime} \partial_{z} T_{p 0}-\frac{T^{\prime}-T_{p}^{\prime}}{\mathrm{St}_{\mathrm{th}}}=0 .
\end{gathered}
$$

In these equations, and in all the relations that follow, time and space coordinates as well as all the field quantities and operators are dimensionless but, to avoid unnecessarily encumbering the writing, we do not use a special notation to indicate this fact.

In addition to the particle Reynolds number $\mathrm{Re}_{p}$ defined in (2.15), the parameters appearing here are the Rayleigh and Prandtl numbers, defined in the same way as in the single-phase case,

$$
\mathrm{Ra}=\frac{g \beta\left(T_{h}-T_{c}\right) H^{3}}{v_{f} D_{f}}, \quad \operatorname{Pr}=\frac{v_{f}}{D_{f}},
$$

the ratio $\delta$ of the particle diameter to the cell height,

$$
\delta=\frac{d_{p}}{H}
$$

the Stokes numbers for momentum and energy,

$$
\mathrm{St}_{m}=\frac{v_{f} \tau_{p}}{H}=\frac{R \delta^{2}}{18 r_{\mathrm{Re}}} \sqrt{\frac{\mathrm{Ra}}{\mathrm{Pr}}}, \quad \mathrm{St}_{\mathrm{th}}=\frac{v_{f} \tau_{\mathrm{th}}}{H}=\frac{\delta^{2} E}{6 \mathrm{Nu}_{p}} \sqrt{\mathrm{RaPr}},
$$

and the physical properties ratios,

$$
R=\frac{\rho_{p}}{\rho_{f}}, \quad E=\frac{\rho_{p} c_{p p}}{\rho_{f} c_{p f}} .
$$

Other than with the Stokes numbers, the effect of the particles appears via the the mass loading and the analogous heat capacity quantity defined by

$$
\mathcal{M}=\frac{\rho_{p}}{\rho_{f}} n_{0} v_{p}=R n_{0} v_{p}, \quad \mathcal{E}=\frac{\rho_{p} c_{p p}}{\rho_{f} c_{p f}} n_{0} v_{p}=E n_{0} v_{p}
$$

Equations (3.13) and (3.15) show that these parameters modulate the effect of the particle Stokes numbers on the fluid. The product $n_{0} v_{p}=\alpha_{0}$ appearing here may be seen as the undisturbed particle volume fraction but, since the present model neglects the volume occupied by the particles, $\alpha_{0}$ should more properly be regarded as a dimensionless form of the undisturbed particle number density. We may also note that

$$
\frac{\mathcal{M}}{\mathrm{St}_{m}}=\frac{18 r_{\mathrm{Re}} n_{0} v_{p}}{\delta^{2}} \sqrt{\frac{\mathrm{Pr}}{\mathrm{Ra}}}, \quad \frac{\mathcal{E}}{\mathrm{St}_{\mathrm{th}}}=\frac{6 \mathrm{Nu}_{p} n_{0} v_{p}}{\delta^{2} \sqrt{\mathrm{RaPr}}} .
$$

The first fraction in the ratio $\mathcal{M} / \mathrm{St}_{m}$ will be recognized as the inverse of the Darcy number $\mathrm{Da}=\kappa / H^{2}$, with the permeability $\kappa$ defined in (2.38). Finally, an additional independent degree 
of freedom is necessary to characterize the temperature at which the particles are introduced at the top boundary. For this purpose we define

$$
T_{p}^{*}=\frac{T_{p t}-T_{c}}{T_{h}-T_{c}} .
$$

The length $\ell$ defined in (2.18) scaled by the particle diameter and the ratio $\ell / L$ appearing in (2.25) for the undisturbed temperature profile are

$$
\frac{\ell}{d_{p}}=\frac{\operatorname{Re}_{p} \operatorname{Pr} E}{6 \mathrm{Nu}_{p}}, \quad \frac{\ell}{L}=\operatorname{Re}_{p} \operatorname{Pr} E \sqrt{\frac{n_{0} v_{p}}{6 \mathrm{Nu}_{p}}} .
$$

The ratio of the particle terminal velocity to the fluid free-fall velocity is

$$
\frac{w_{0}}{v_{f}}=\frac{\operatorname{Re}_{p}}{\delta} \sqrt{\frac{\mathrm{Pr}}{\mathrm{Ra}}} .
$$

\section{NUMERICAL SOLUTION}

The solution of Eqs. (3.13) to (3.16) is sought in the form of normal modes with a time dependence proportional to $\exp (\lambda t)$, with the $\lambda$ 's eigenvalues of the spatial operator. The secondand fourth-order spatial derivatives are discretized by central differences except for the term $\partial_{z}^{2} w_{z}^{\prime}$ at the node next to the bottom boundary where the lack of a boundary condition for $w_{z}^{\prime}$ forces us to use a second-order accurate one-sided derivative. First-order accurate upwind differencing was used for the first-order derivatives in the convective terms in (3.14) and (3.16). We ran several simulations with both 80 and 120 nodes to examine grid convergence finding essentially indistinguishable results.

The generalized eigenvalue problem resulting from the discretization was solved using the function eig $(\cdot, \cdot)$ of MATLAB based on the generalized Schur decomposition of the matrices. Even though the spatial operator of the problem is not self-adjoint, we found that the dominant modes have real eigenvalues. For each wave number $k$ we increased the Rayleigh number starting from an appropriately small value until the real part of the dominant eigenvalue became positive and repeated the search by gradually increasing $k$. The critical Rayleigh number reported in the figures in the next section is the smallest value of Ra identified in this way, typically with a relative accuracy of $0.1 \%$ or better.

\section{NUMERICAL RESULTS}

The introduction of particles greatly increases the number of dimensionless parameters necessary to characterize the flow. Summarizing, in addition to the Rayleigh and Prandtl numbers Ra and Pr, a minimal set of parameters would include the undisturbed particle volume fraction $\alpha_{0}$, the particle Reynolds number $\mathrm{Re}_{p}$, the ratio $\delta$ of the particle diameter to the cell height, the property ratios $R$ and $E$, and the dimensionless temperature of the injected particles, $T_{p}^{*}=\left(T_{p t}-T_{c}\right) /\left(T_{h}-T_{c}\right)$. In addition to their number, another factor that makes it difficult or impossible to isolate the effects of specific parameters, each one of which would, by itself, permit a focus on specific physical effects, is the mutual relationships among them. For example, the ratio of the two Stokes numbers is

$$
\frac{\mathrm{St}_{m}}{\mathrm{St}_{\mathrm{th}}}=\frac{\mathrm{Nu}_{p}}{3 r_{\mathrm{Re}}} \frac{R}{\operatorname{Pr} E}
$$

The first factor depends (rather weakly, in the range of present concern) on the particle Reynolds number, while the second group will be fixed once the physical properties of the fluid and particles are specified. Thus, $\mathrm{St}_{m}$ and $\mathrm{St}_{\mathrm{th}}$ cannot be varied independently without changing other parameters. As can be seen from (2.15), for a given kinematic viscosity, i.e., a given fluid, the particle Reynolds number depends on the particle-fluid density ratio $R$ and on the particle diameter. Thus, for a 


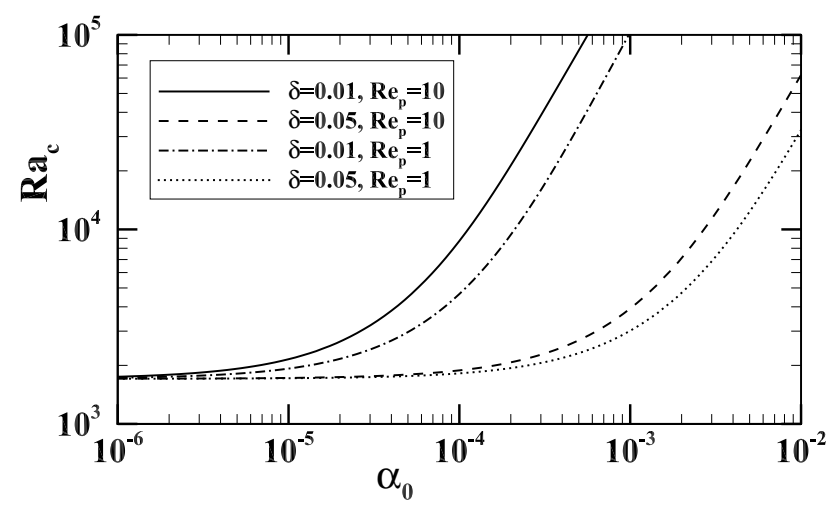

FIG. 2. Critical Rayleigh number vs undisturbed particle volume fraction $\alpha_{0}$ for the droplets/air case. The parameter values are $R=800, E=3385, \operatorname{Pr}=0.71, T_{p}^{*}=0$.

given $\delta=d_{p} / H$, the Reynolds number can be changed either by changing the density ratio, which will affect the mechanical Stokes number, or by changing the cell height $H$, which will affect the Rayleigh number. The mass and thermal loadings depend on $R$ and $E$, the latter dependent on $\mathrm{St}_{\text {th }}$ once $\delta, \operatorname{Re}_{p}, \operatorname{Pr}$, and $\operatorname{Ra}$ are specified. Given the intricacies of this situation, the best we can do is to show some results useful to illustrate broad trends. We focus on two particular cases, one, with $R=800, E=3385, \operatorname{Pr}=0.71$, representative of a water-droplet/air system, and the other, with $R=3, E=0.5$, and $\operatorname{Pr}=5$, representative of a sand/water system.

A general feature of all the results that follow is that particles have a stabilizing effect, as can be understood from the similarity pointed out earlier with Rayleigh-Bénard convection in a porous medium. The effect is quite strong when the density of the disperse phase is much larger than that of the fluid as can be seen in Fig. 2 for the droplets/air case, particularly when the particle diameter is small. Although in all cases the critical Rayleigh number starts close to the single-phase value $\mathrm{Ra}=1707$ when the particle volume fraction $\alpha_{0}$ is small, it quickly rises with increasing $\alpha_{0}$, the faster the smaller $\delta$ and the larger $\operatorname{Re}_{p}$. Figure 3 is analogous for the sand/water system, with the particles introduced at the cold-plate temperature $T_{p}^{*}=0$ as in the previous figure, and again with $\delta=0.01$ and 0.05 , and $\operatorname{Re}_{p}=1$ and 10. In this case the effect of the particles takes longer to manifest itself, so much so that the case with $\delta=0.01$ and $\operatorname{Re}_{p}=1$ remains indistinguishable

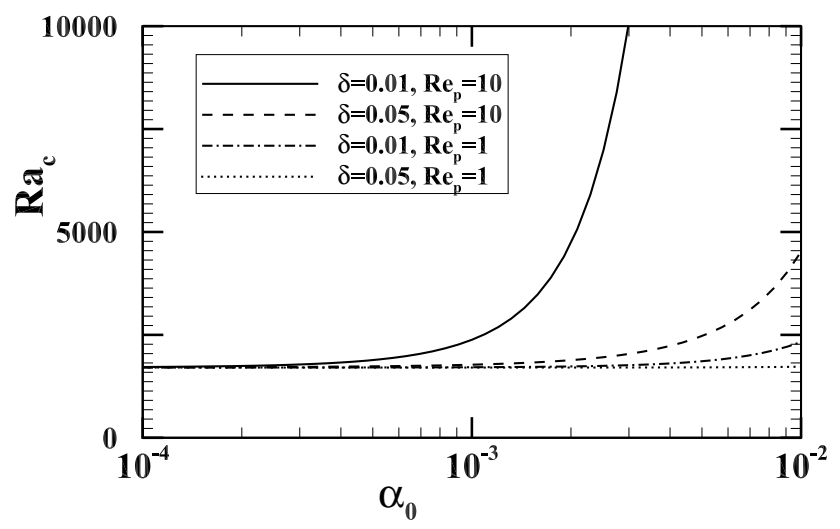

FIG. 3. Critical Rayleigh number vs undisturbed particle volume fraction $\alpha_{0}$ for the sand/water case. The parameter values are $R=3, E=0.5, \operatorname{Pr}=5, T_{p}^{*}=0$. 


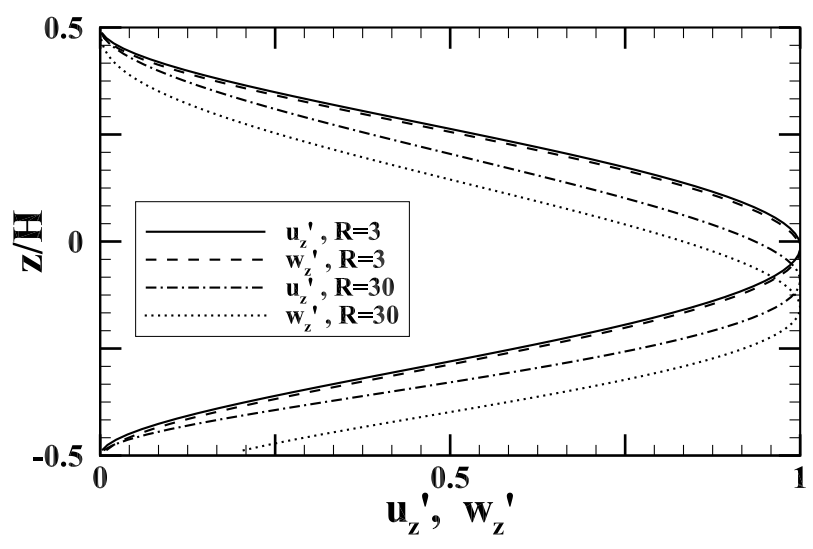

FIG. 4. Examples of the velocity eigenvectors corresponding to the $\lambda=0$ eigenvalue for two difference ratios, $R=3$ and $R=30$. The other parameters are $\alpha_{0}=0.01, \delta=0.05, \operatorname{Re}_{p}=1, E=0.15 R, T_{p}^{*}=0, \operatorname{Pr}=5$.

from the single-phase system even at a volume fraction of $1 \%$ at which the droplet/air system is already considerably influenced by the disperse phase. This is a general feature of systems in which the density contrast is not very marked. Contrary to the previous case, this system appears to be affected more by the increase of $\delta$ from 0.01 to 0.05 than by the increase of $\operatorname{Re}_{p}$ from 1 to 10 .

The stabilizing effect of increasing $R$ is easily understood from the two momentum equations. The coefficient of the last term of (3.13) is independent of $R$ and, therefore, the stabilizing effect of this term will mostly depend on the magnitude of the velocity difference $u_{z}^{\prime}-w_{z}^{\prime}$. As is evident from the particle momentum equation (3.14), the particle velocity perturbation is a consequence of drag by the fluid, so that $\left|u_{z}^{\prime}-w_{z}^{\prime}\right|<\left|u_{z}^{\prime}\right|$. Since particle inertia increases with $R$, the effectiveness of drag in reducing the difference $u_{z}^{\prime}-w_{z}^{\prime}$ decreases. The last term of (3.14) gains thereby importance, which stabilizes the flow. [Indeed we have found that setting $w_{z}^{\prime}=0$, which increases the importance of the last term of (3.14), has a marked stabilizing effect.] This explanation is made evident by the eigenvectors corresponding to the eigenvalue with the largest real part $\lambda=0$, examples of which are shown in Fig. 4 for $R=3$ and $R=30$. When the density contrast is small, the fluid and particle eigenvectors are close to each other, but a significant difference appears for $R=30$.

Increasing the particle Reynolds number has a stabilizing effect for the same reason as increasing $R$. Increasing $\delta$ can be seen as amounting to increasing the particle response time $\tau_{p}$ and, therefore, the effect on the particle momentum is similar as can be seen comparing the two coefficients in (3.14) which are the only ones that matter since the time derivative term vanishes at marginal conditions.

For $\delta=0.05$, the dimensionless wave number $k H$ corresponding to the critical Rayleigh number appears to be little affected by the particle volume fraction in the range shown in Figs. 2 and 3. For example, for the sand/water case, $k H$ grows from $\sim 3.1$ to $\sim 3.25$ for $\operatorname{Re}_{p}=1$ and between $\sim 3.1$ and $\sim 3.7$ for $\operatorname{Re}_{p}=10$. For $\delta=0.01$, however, the dependence is stronger, increasing from $\sim 3$ up to $\sim 10$ for both Figs. 2 and 3; an example for the former case is shown in Fig. 5. For the sand/water system, an increase of this magnitude requires a much larger volume fraction. A plausible explanation for this result is that the mean interparticle distance is of the order of $d_{p} / \alpha_{0}^{1 / 3}$ or, in dimensionless form, $\delta / \alpha_{0}^{1 / 3}$, a fact that introduces smaller and smaller spatial scales as the particle volume fraction is increased.

Figures 6 and 7 show the effect of the particle temperature for $\delta=0.01$ and the same droplets/air and sand/water systems, with $\alpha_{0}=10^{-4}$ and $\operatorname{Re}_{p}=10$ for the former and $\alpha_{0}=0.01$ and $\operatorname{Re}_{p}=1$ for the latter. Since, as shown by (3.15), the particles act as a heat source for the system, increasing their temperature would be expected to enhance stability by decreasing the degree of stratification, which is indeed what is observed for the sand/water system and, in the first part of the temperature 


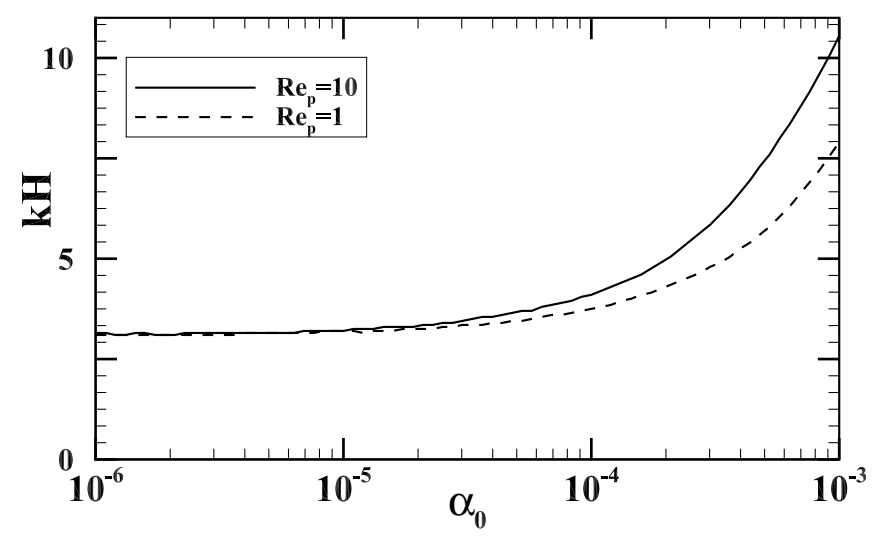

FIG. 5. The dimensionless wave number $k H$ corresponding to the critical Rayleigh number of the droplets/air case shown in Fig. 2. The parameter values are $R=3, \delta=0.01, E=0.5, \operatorname{Pr}=5, T_{p}^{*}=0$.

range, for the droplets/air system as well. In the latter case, however, the critical Rayleigh number reaches a maximum and then starts to decrease with further increasing the particle temperature, a phenomenon that is also encountered for the other system at larger values of $T_{p}^{*}$. The explanation for this inversion lies in the effect that the particle temperature has on the undisturbed temperature distribution shown earlier in Fig. 1. As long as the temperature distribution is monotonically decreasing with height, warmer fluid is transported upward by the fluid disturbance velocity in the regions where $u_{z}^{\prime}>0$ while colder fluid is transported downward where $u_{z}^{\prime}<0$. Both effects are destabilizing as, being lighter, the warmer fluid will tend to rise further and the colder fluid, conversely, to sink deeper. Thus, the system is stabilized if the vertical temperature gradient becomes less negative, which is a consequence of a moderate increase of $T_{p}^{*}$ as can be seen comparing the temperature distributions for $T_{p}^{*}=0$ and $T_{p}^{*}=1$ in Fig. 1 . This is the cause of the increasing critical Rayleigh number with $T_{p}^{*}$ in part of Fig. 6 and in Fig. 7. However, by inducing a nonmonotonic undisturbed temperature distribution in the cell, a further increase of $T_{p}^{*}$ creates a strongly negative temperature gradient in the upper part of the cell, which favors instability, and the critical Rayleigh number starts to decrease. The undisturbed temperature is nonmonotonic also for $T_{p}^{*}=-1$, but the region where it is growing with height is smaller than that where it is strongly decreasing and this

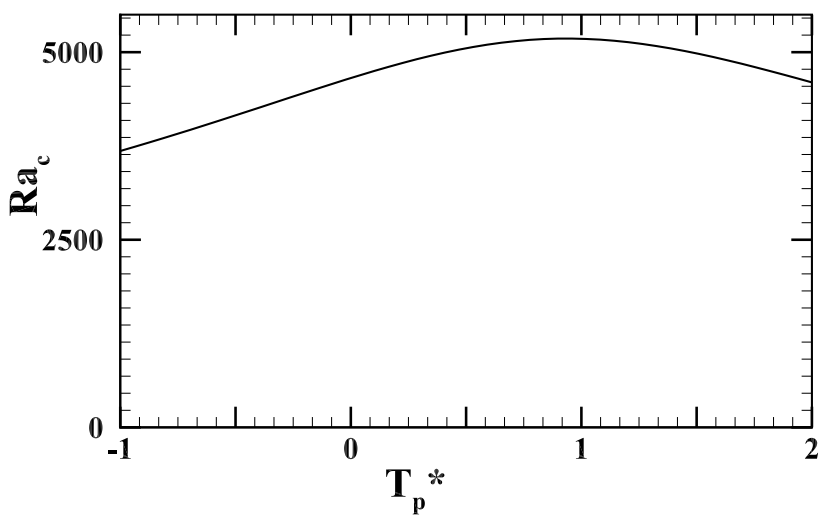

FIG. 6. Effect of the normalized particle temperature $T_{p}^{*}=\left(T_{p t}-T_{c}\right) /\left(T_{h}-T_{c}\right)$ on the critical Rayleigh number for the droplets/air system with $\delta=0.01, R=800, E=3385, \operatorname{Pr}=0.71, \operatorname{Re}_{p}=1$, and $\alpha_{0}=10^{-4}$. Note the lack of monotonicity as $T_{p}^{*}$ is increased. 


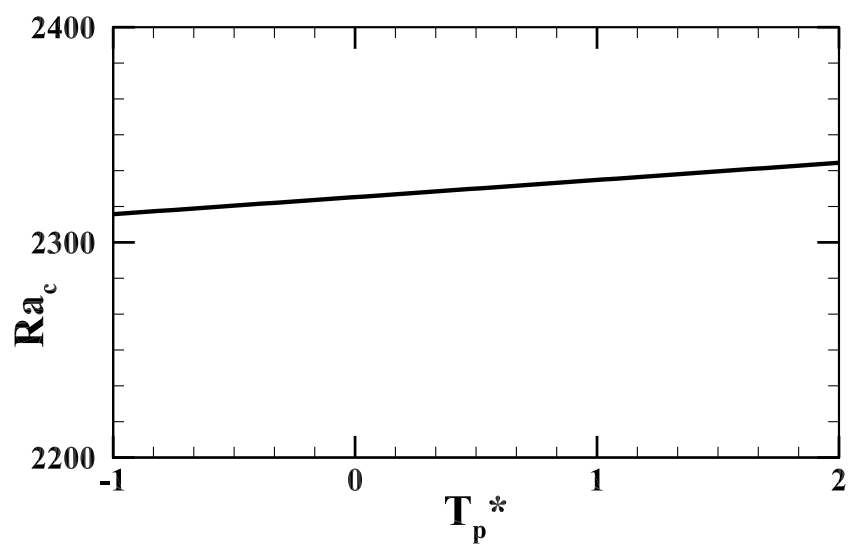

FIG. 7. Effect of the normalized particle temperature $T_{p}^{*}=\left(T_{p t}-T_{c}\right) /\left(T_{h}-T_{c}\right)$ on the critical Rayleigh number for the sand/water system with $\delta=0.01, R=3, E=0.5, \operatorname{Pr}=5, \operatorname{Re}_{p}=1$, and $\alpha_{0}=0.01$.

latter region prevails. Due to the difference in physical properties, in the case of the sand-water system the undisturbed temperature becomes nonmonotonic only for larger values of $T_{p}^{*}$ which are somewhat unrealistic and, therefore, are not included in the range of $T_{p}^{*}$ values covered in Fig. 7. We have verified however that the critical Rayleigh number starts to decrease for sufficiently large values of $T_{p}^{*}$ also for this system.

Figure 8 shows an example of the dependence of the stability threshold on the mechanical Stokes number, varied by varying the ratio $R$ of the particles-to-fluid densities. Here $\delta=0.05, \alpha_{0}=0.01$, $c_{p p} / c_{p f}=0.15, \operatorname{Pr}=5$, and $\operatorname{Re}_{p}=1$. In descending order, the lines correspond to $T_{p}^{*}=1,0$, and -1 . As $\mathrm{St}_{m}$ increases with $R$, the fluid-particle velocity difference increases as explained before, which stabilizes the system. As shown in Fig. 1, warm particles, with $T_{p}^{*}=1$, reduce the undisturbed temperature gradient in the cell and therefore produce a stronger stabilizing effect. On the other hand, very cold particles, with $T_{p}^{*}=-1$, greatly increase the negative temperature gradient in the lower part of the cell, which diminishes their stabilizing influence.

An example of the effect of the thermal Stokes number $\mathrm{St}_{\mathrm{th}}$, varied by varying the heat capacity ratio $E$, is shown in Fig. 9 for the droplets/air (solid line) and the sand/water systems. Here $\delta=0.05, \alpha_{0}=0.01, T_{p}^{*}=0$, and $\operatorname{Re}_{p}=1$. The general trend is similar to that seen before in Fig. 8

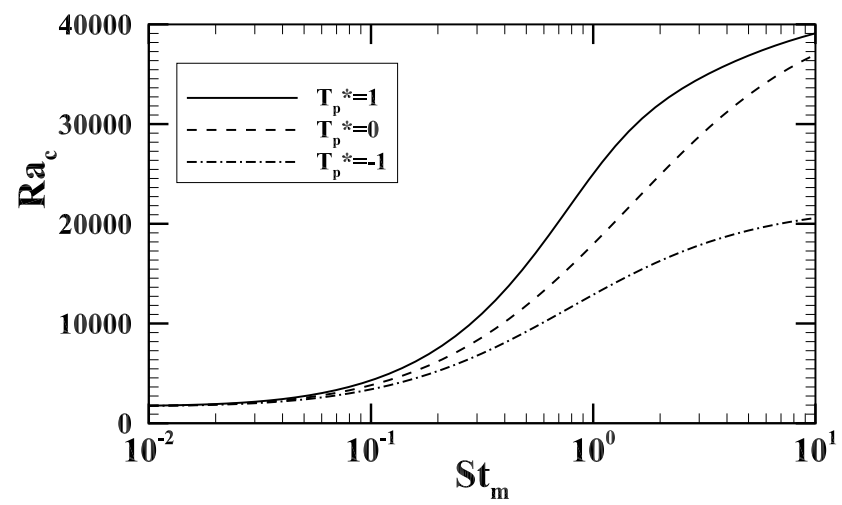

FIG. 8. Effect of the particle mechanical Stokes number $\mathrm{St}_{m}$ (varied by varying the density ratio $R$ ) on the stability threshold for the sand/water case. In descending order, the particle injection temperature is $T_{p}^{*}=1$, 0 , and -1 . The parameter values are $\delta=0.05, \alpha_{0}=0.01, c_{p p} / c_{p f}=0.15, \operatorname{Pr}=5, \operatorname{Re}_{p}=1$. 


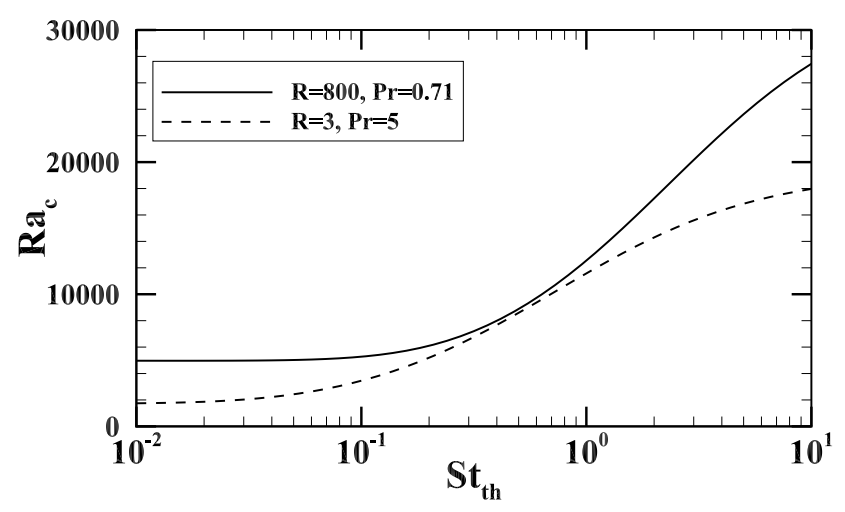

FIG. 9. Effect of the particle thermal Stokes number $\mathrm{St}_{\mathrm{th}}$ (varied by varying the heat capacity ratio $E$ ) on the stability threshold for the droplets/air (solid line) and sand/water cases with $\alpha_{0}=0.01$. The parameter values are $\delta=0.05, T_{p}^{*}=0$, and $\operatorname{Re}_{p}=1$.

when the density ratio $R$ is varied, and for a similar reason. Indeed, the ratio $\mathcal{E} / \mathrm{St}_{\text {th }}$ in the fluid energy equation (3.15) is independent of $E$, while a large $E$, or a large $\mathrm{St}_{\mathrm{th}}$, hinders the equalization of the particle and fluid temperatures. This increases the difference $T^{\prime}-T_{p}^{\prime}$ in the fluid energy equation and, with it, the effective energy source, which tends to destabilize the system. This explanation is confirmed by the appearance of the eigenvectors, examples of which are shown in Fig. 10. Analogously to Fig. 4, we see that the temperature difference is small for the sand/water case, for which $E=0.5$, while it becomes very large for the droplet/air system for which $E=3385$.

\section{SUMMARY AND CONCLUSIONS}

This paper started with the presentation of a mathematical model of the two-fluid type for the study of the Rayleigh-Bénard convection in a laterally unbounded cell with the bottom plate kept at a higher temperature than the top one. The general model was then specialized to the situation in which particles are introduced steadily and uniformly at the top plate at their terminal velocity. The effect of the particles on the stability threshold of the system was investigated by a standard decomposition into normal modes.

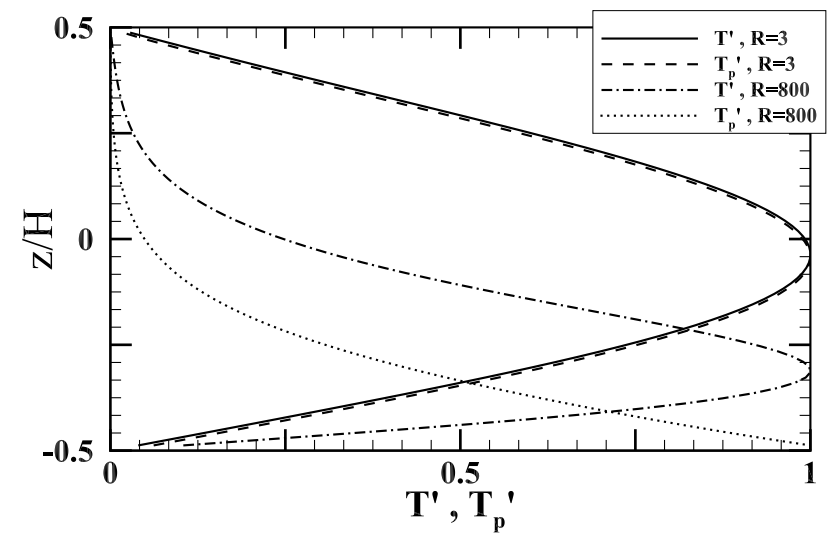

FIG. 10. Examples of the temperature eigenvectors for the droplet/air and sand/water system for $\alpha_{0}=$ 0.01 . The other parameters are $\delta=0.05, T_{p}^{*}=0, \operatorname{Re}_{p}=1$. 
The addition of particles greatly enlarges the parameter space of the problem and it has not been possible to carry out an exhaustive parameter study. Rather, we have focused on two specific cases in which the physical properties are similar to those of a sand/water system and to those of a water-droplets/air system. The numerical results have shown that particles tend to inhibit the onset of natural convection thereby stabilizing the system. This effect can be understood noting that the particles resemble a distributed drag force and heat source in the fluid, similarly to a porous medium. An interesting result was found to occur when the particles are injected at a temperature higher than that of the bottom plate. In this case the undisturbed temperature distribution ceases to be monotonic with the effect that, all other parameters being the same, the system is destabilized.

Other situations can be studied within the framework of the mathematical model outlined in Sec. II. For example, it may be expected that if particles are introduced nonuniformly at the top plate, or with a variable velocity, or with unequal density, they would promote, rather than hinder, the onset of convection. A similar but particularly interesting question due to the competition between physical processes would be the effect of introducing particles at different temperatures. These problems will be investigated in future work.

\section{ACKNOWLEDGMENTS}

The authors are grateful to Prof. Detlef Lohse for some useful comments. This work has been supported by the US National Science Foundation under Grant No. CBET 2053204.

[1] S. Balachandar and J. K. Eaton, Turbulent dispersed multiphase flow, Annu. Rev. Fluid Mech. 42, 111 (2010).

[2] J. G. M. Kuerten, Point-particle DNS and LES of particle-laden turbulent flow: A state-of-the-art review, Flow Turb. Comb. 97, 689 (2016).

[3] M. Maxey, Simulation methods for particulate flows and concentrated suspensions, Annu. Rev. Fluid Mech. 49, 171 (2017).

[4] D. A. Nield and A. V. Kuznetsov, Thermal instability in a porous medium layer saturated by a nanofluid, Int. J. Heat Mass Transfer 52, 5796 (2009).

[5] D. A. Nield and A. V. Kuznetsov, The effect of local thermal nonequilibrium on the onset of convection in a nanofluid, J. Heat Transfer 132, 052405 (2010).

[6] D. A. Nield and A. V. Kuznetsov, The onset of convection in a horizontal nanofluid layer of finite depth, Eur. J. Mech. B/Fluids 29, 217223 (2010).

[7] D. A. Nield and A. V. Kuznetsov, The onset of convection in a horizontal nanofluid layer of finite depth: A revised model, Int. J. Heat Mass Transfer 77, 915 (2014).

[8] J. Buongiorno, Convective transport in nanofluids, J. Heat Transfer 128, 240 (2006).

[9] Z. Haddad, H.-F. Oztop, E. Abu Nada, and A. Mataoui, A review on natural convective heat transfer of nanofluids, Renewable Sustainable Energy Rev. 16, 5363 (2012).

[10] S. Javed, H. M. Ali, H. Babar, M. S. Khan, M. M. Janjua, and M. A. Bashir, Internal convective heat transfer of nanofluids in different flow regimes: A comprehensive review, Phys. A: Stat. Mech. Appl. 538, 122783 (2020).

[11] O. Mahian, L. Kolsi, M. Amani, P. Stelle, G. Ahmadi, C. Kleinstreuer, J. S. Marshalli, M. Siavashi, R. A. Taylor, H. Niazmand, S. Wongwises, T. Hayat, A. Kolanjiyil, A. Kasaeian, and L. Pop, Recent advances in modeling and simulation of nanofluid flows, Part I: Fundamentals and theory, Phys. Rep. 790, 1 (2019).

[12] J. C. Gu, S. Takeuchi, and T. Kajishima, Influence of Rayleigh number and solid volume fraction in particle-dispersed natural convection, Int. J. Heat Mass Transfer 120, 250 (2018).

[13] T. Kajishima, Immersed boundary/solid method for the numerical simulation of particle-laden flows, Fluid Dyn. Res. 51, 051401 (2019).

[14] S. Takeuchi, Y. Miyamori, J. C. Gu, and T. Kajishima, Flow reversals in particle-dispersed natural convection in a two-dimensional enclosed square domain, Phys. Rev. Fluids 4, 084304 (2019). 
[15] S. Takeuchi, T. Tsutsumi, K. Kondo, T. Harada, and T. Kajishima, Heat transfer in natural convection with finite-sized particles considering thermal conductance due to inter-particle contacts, Comput. Therm. Sci. 7, 385 (2015).

[16] K. Walayat, Z. L. Zhang, K. Usman, J. Z. Chang, and M. B. Liu, Dynamics of elliptic particle sedimentation with thermal convection, Phys. Fluids 30, 103301 (2018).

[17] P. Oresta, F. Fornarelli, and A. Prosperetti, Multiphase Rayleigh-Bénard convection, Mech. Eng. Rev. 1, FE0003 (2014).

[18] P. Oresta and A. Prosperetti, Effects of particle settling on Rayleigh-Bénard convection, Phys. Rev. E 87, 063014 (2013).

[19] H. J. Park, K. O'Keefe, and D. H. Richter, Rayleigh-Bénard turbulence modified by two-way coupled inertial, nonisothermal particles, Phys. Rev. Fluids 3, 034307 (2018).

[20] V. Patocka, E. Calzavarini, and N. Tosi, Settling of inertial particles in turbulent Rayleigh-Bénard convection, Phys. Rev. Fluids 5, 114304 (2020).

[21] A. Xu, S. Tao, L. Shi, and H.-D. Xi, Transport and deposition of dilute microparticles in turbulent thermal convection, Phys. Fluids 32, 083301 (2020).

[22] B. Gereltbyamba and C. Lee, Behavior of settling inertial particles in a differentially heated cubic cavity at moderate Rayleigh number, J. Mech. Sci. Technol. 32, 3169 (2018).

[23] B. Gereltbyamba and C. Lee, Flow modification by inertial particles in a differentially heated cubic cavity, Int. J. Heat Fluid Flow 79, 108445 (2019).

[24] R. Puragliesi, A. Dehbi, E. Leriche, A. Soldati, and M. O. Deville, DNS of buoyancy-driven flows and Lagrangian particle tracking in a square cavity at high Rayleigh numbers, Int. J. Heat Fluid Flow 32, 915 (2011).

[25] M. Lappa, On the transport, segregation, and dispersion of heavy and light particles interacting with rising thermal plumes, Phys. Fluids 30, 033302 (2018).

[26] A. Frankel, H. Pouransari, F. Coletti, and A. Mani, Settling of heated particles in homogeneous turbulence, J. Fluid Mech. 792, 869 (2016).

[27] H. Pouransari and A. Mani, Effects of preferential concentration on heat transfer in particle-based solar receivers, J. Solar Energy Eng. 139, 021008 (2017).

[28] M. Rahmani, G. Geraci, G. Iaccarino, and A. Mani, Effects of particle polydispersity on radiative heat transfer in particle-laden turbulent flows, Int. J. Multiphase Flow 104, 42 (2018).

[29] S. Chandrasekhar, Hydrodynamic and Hydromagnetic Stability (Clarendon, Oxford, 1961; reprinted by Dover, 1981).

[30] A. Barletta, Routes to Absolute Instability in Porous Media, 1st ed. (Springer, Cham, 2019).

[31] D. A. Nield and A. Bejan, Convection in Porous Media, 5th ed. (Springer, Cham, 2017).

[32] A. W. Woods, Flow in Porous Rocks (Cambridge University Press, Cambridge, 2015).

[33] H. Emami-Meybodi, H. Hassanzadeh, C. P. Green, and J. Ennis-King, Convective dissolution of $\mathrm{CO}_{2}$ in saline aquifers: Progress in modeling and experiments, Int. J. Greenhouse Gas Control 40, 238 (2015).

[34] H. E. Huppert and J. A. Neufeld, The fluid mechanics of carbon dioxide sequestration, Annu. Rev. Fluid Mech. 46, 255 (2014).

[35] S. Mahmoodpour, B. Rostami, M. R. Soltanian, and M. A. Amooie, Convective Dissolution of Carbon Dioxide in Deep Saline Aquifers: Insights from Engineering a High-Pressure Porous Visual Cell, Phys. Rev. Appl. 12, 034016 (2019).

[36] A. Rabinovich, K. Itthisawatpan, and L. J. Durlofsky, Upscaling of $\mathrm{CO}_{2}$ injection into brine with capillary heterogeneity effects, J. Pet. Sci. Eng. 134, 60 (2015).

[37] I. Ataei-Dadavi, M. Chakkingal, S. Kenjeres, C. R. Kleijn, and M. J. Tummers, Flow and heat transfer measurements in natural convection in coarse-grained porous media, Int. J. Heat Mass Transfer 130, 575 (2019).

[38] D. R. Hewitt, Vigorous convection in porous media, Proc. R. Soc. A 476, 20200111 (2020).

[39] D. R. Hewitt, J. A. Neufeld, and J. R. Lister, High Rayleigh number convection in a three-dimensional porous medium, J. Fluid Mech. 748, 879 (2014). 
[40] S. Liu, L. Jiang, K. L. Chong, X. Zhu, Z.-H. Wan, R. Verzicco, R. J. A. M. Stevens, D. Lohse, and C. Sun, From Rayleigh-Bénard convection to porous-media convection: How porosity affects heat transfer and flow structure, J. Fluid Mech. 895, A18 (2020).

[41] S. Elghobashi and G. C. Truesdell, On the 2-way interaction between homogeneous turbulence and dispersed solid particles. I: Turbulence modification, Phys. Fluids A 5, 1790 (1993).

[42] F. E. Marble, Dynamics of dusty gases, Annu. Rev. Fluid Mech. 2, 397 (1970).

[43] M. R. Maxey, The gravitational settling of aerosol-particles in homogeneous turbulence and random flowfields, J. Fluid Mech. 174, 441 (1987).

[44] S. Elghobashi, Direct numerical simulation of turbulent flows laden with droplets or bubbles, Annu. Rev. Fluid Mech. 51, 217 (2019).

[45] W. D. Fullmer and C. M. Hrenya, The clustering instability in rapid granular and gas-solid flows, Annu. Rev. Fluid Mech. 49, 485 (2017).

[46] A. Bejan, Convection Heat Transfer, 4th ed. (Wiley, New York, 2013).

[47] R. Clift, J. R. Grace, and M. E. Weber, Bubbles, Drops, and Particles (Academic Press, New York, 1978; reprinted by Dover, 2005).

[48] T. L. Bergman, A. S. Lavine, F. P. Incropera, and D. P. De Witt, Fundamentals of Heat and Mass Transfer, 8th ed. (Wiley, Hoboken, NJ, 2018).

[49] W. E. Ranz and W. R. Marshall, Evaporation from drops, Chem. Eng. Prog. 48, 141 (1952).

[50] G. Ahlers, S. Grossman, and D. Lohse, Heat transfer and large-scale dynamics in turbulent RayleighBénard convection, Rev. Mod. Phys. 81, 503 (2009).

[51] D. Lohse and F. Toschi, The Ultimate State of Thermal Convection, Phys. Rev. Lett. 90, 034502 (2003).

[52] A. Aliseda, A. Cartellier, F. Hainaux, and J. C. Lasheras, Effect of preferential concentration on the settling velocity of heavy particles in homogeneous isotropic turbulence, J. Fluid Mech. 468, 77 (2002). 